\title{
A alfabetização científica em nutrição humana: intersecções e divergências
}

Scientific literacy in human nutrition: intersections and divergences

\author{
Valéria Brumaro Regina Fornazari ${ }^{1}$ \\ Ana Tyiomi Obara²
}

\section{Resumo}

Este artigo tem como objetivo apresentar uma revisão bibliográfica sobre a alfabetização em nutrição como uma probabilidade para o ensino de ciências. Para tanto, foi realizada uma extensa busca nas principais bases de dados nacionais e internacionais partindo das palavras chave - em língua portuguesa, inglesa, francesa e espanhola - alfabetização científica, alfabetização em nutrição e alfabetização em saúde revelando que a alfabetização em nutrição, enquanto perspectiva, tem como fundamentos possibilitar ao indivíduo desenvolver autonomia e criticidade em relação aos conhecimentos de nutrição de forma a tomar decisões individuais e coletivas quanto a formação de hábitos alimentares e qualidade de vida.

Palavras chave: alimentação; ensino de aprendizagem; estado da arte.

\section{Abstract}

This article aims to present a literature review on nutrition literacy as a probability for the teaching of science. Therefore, an extensive search was performed in the main national and international databases. The key words used were - in Portuguese, English, French and Spanish - scientific literacy, nutrition literacy and health literacy, revealing that literacy in nutrition, has as basis to enable the individual to develop autonomy and criticality in relation to nutrition knowledge in order to make individual and collective decisions regarding the formation of eating habits and quality of life.

Keywords: food; learning teaching; state of art.

\section{Introdução}

$\mathrm{Na}$ atualidade fala-se muita na necessidade de o cidadão ser alfabetizado cientificamente, ou seja, ter acesso ao conhecimento científico podendo atuar efetivamente na sociedade, além de tomar decisões, pessoais e coletivas, de forma consciência. Porém, esta discussão não é recente, de acordo com DeBoer (2000), o termo alfabetização científica, traduzido no inglês scientific literacy, data de meados da década de 1950, apresenta diferentes definições, conforme os vários autores, o que torna um problema quando esta proposta é o objetivo da reforma da educação científica. Segundo o mesmo

\footnotetext{
${ }^{1}$ Universidade Estadual de Maringá | nutri.valeria.regina@gmail.com

${ }^{2}$ Universidade Estadual de Maringá | anatobara@gmail.com
} 
autor, houveram inúmeras tentativas em definir alfabetização científica, porém ainda não é possível afirmar que exista uma definição única aceita pelos profissionais da área da educação. Isso se deve, de acordo com Good, Hafner and Pebbles (2000) e DeBoer (2000), pelo fato das definições serem constantemente refeitas à medida que a própria ciência progride. Ainda, para DeBoer (1997), a alfabetização científica tem se apresentado como um slogan motivador que justifique implementos para a melhoria do ensino e aprendizagem das ciências, portanto, falar em alfabetização científica seria o mesmo que falar em educação científica.

Nesta perspectiva, esta terminologia tem sido transposta por outras áreas, como a Saúde e a Informática, gerando uma infinidade de significados, o que, infelizmente não modificam a realidade, deixando de atender as finalidades as que se propõem. No que tange a área de Saúde, é notório que as maiores causas de mortes no mundo decorrem de doenças que poderiam ser prevenidas e tratadas com atitudes como alimenta-se bem, praticar atividades físicas, controlar o estresse, ter uma rotina equilibrada entre o trabalho e a atividades de lazer, ou seja, apresentar hábitos de vida saudável (RODRIGUES; RONCADA, 2008). Assim, emerge nesse cenário a proposta da alfabetização em saúde na qual a alfabetização em nutrição e alimentação estão contidas. Estas propostas buscam integrar os conhecimentos das Ciências da Saúde na formação humana promovendo a auto-gestão das decisões individuais e coletivas em prol da melhora da qualidade de vida. Entretanto, é inevitável questionar como essas propostas podem se efetivar em transformações? Quais são suas inter-relações e influencias no processo de ensino de aprendizagem?

Desta forma, entendemos estas como reflexões necessárias e importantes para o gerenciamento real de uma reforma eficiente e promissora do Ensino das Ciências na Educação Básica. Por conseguinte, este artigo objetiva apresentar uma revisão bibliográfica acerca da alfabetização científica, da alfabetização em saúde e em nutrição de alimentação, encontrando seus pontos de convergência e divergência de forma a identificar a possibilidade e limites destes nos processos de ensino e aprendizagem de Ciências na Educação Básica.

\section{A oscilação da terminologia}

De acordo com Sasseron e Carvalho (2011), na didática das ciências, existem inúmeros termos que se preocupam com a formação cidadã. Na língua espanhola, por exemplo, utiliza-se "Alfabetizácion Científica" enquanto que em inglês encontramos "Scientific Literacy" e na língua francesa "Alphabétisation Scientifique". Estes termos têm em comum promover o ensino de Ciências pautado na compreensão do conhecimento científico e de suas interrelações com o mundo de forma a possibilitar ao educando a mobilizações destas ideias para a tomada de decisão diária, tanto no âmbito individual como coletivo.

Contudo, quando realizamos uma revisão bibliográfica acerca desta perspectiva de ensino nos deparamos com terminologias distintas. Esta situação ocorre no momento em que as terminologias acimas citadas são traduzidas. Quando esta tradução ocorre partindo da língua espanhola e francesa utilizamos o termo "Alfabetização Científica" e quando parte da língua inglesa, definimos como "Letramento Científico" (SASSERON; CARVALHO 2011).

Uma outra terminologia utilizada é "Enculturação Científica". Alguns autores, como Carvalho e Tinoco (2006), utilizam a terminologia como sendo um sinônimo de 
alfabetização científica. Já para outros como Mortimer e Machado (1996), a enculturação científica se dá por meio da aprendizagem da cultura científica.

Diante do exposto, podemos perceber que, na língua portuguesa a terminologia se apresenta se forma difusa e dinâmica, com vários significados, o que também ocorre em outras línguas (DEBOER, 2000; LAUGKSCH, 1999). Assim, neste trabalho, objetivando a padronização da terminologia, foi utilizada a expressão Alfabetização Científica, que será caracterizada ao longo da revisão bibliográfica apresentada.

\section{A Alfabetização científica: um resgate histórico}

Por conta das flutuações semânticas e pelo dinamismo dos conceitos envolvidos na caracterização da alfabetização científica, será apresentada uma revisão bibliográfica a partir dos termos "Alfabetizácion Científica", "Scientific Literacy", "Alphabétisation Scientifique", "Educação Científica", "Alfabetização Científica", "Letramento Científico" e "Enculturação Científica". Ressaltamos que, de acordo com Sasseron e Carvalho (2011), as expressões apresentadas são utilizadas, preferencialmente, por alguns escritores consagrados da área de ensino de Ciências tais como: Mamede e Zimmermann (2007), Santos e Mortimer (2001) que adotam Letramento Científico, Brandi e Gurgel (2002), Auler e Delizoicov (2001), Lorenzetti e Delizoicov (2001), Chassot (2000) que utilizam Alfabetização científica e Carvalho e Tinoco (2006), Mortimer e Machado (1996) que usam Enculturação Científica.

Entretanto, apesar da variação semântica, as expressões são convergentes nos aspectos essenciais da proposta quanto a necessidade de o ensino de Ciências promover a formação crítica, reflexiva e autônoma dos educandos de forma a relacionar os conhecimentos escolares com o dia a dia destes, destacando as relações existentes entre a ciência, a tecnologia, a sociedade e o ambiente (CST). Desta forma, a presente revisão não tem como objetivo o enfoque semântico, mas sim epistemológico em elucidar e caracterizar a expressão adotada.

Inicialmente buscamos pelos primeiros trabalhos publicados. Provavelmente a primeira citação do termo scientific literacy foi realizada pela fundação Rockefeller Brothers, em 1958, ao pulicar um relatório intitulado "Prospect for America: the problems and opportunities confronting American democracy - in foreign polity, in military preponderance, in education, in social and economic affairs", no qual foi discutida a qualidade da educação estadunidense, evidenciando a preocupação da comissão em como o sistema educacional poderia preparar os indivíduos para viver e trabalhar em um mundo tecnológico permeado por rápidas mudanças. Dentre as estratégias propostas, a alfabetização científica foi sinalizada - "assim como nós devemos insistir que cada cientista seja amplamente educado, também devemos insistir que cada pessoa educada seja alfabetizada em ciência" (LEAR, 1958, p.261, tradução nossa).

Nesse mesmo período e pautado nas mesmas preocupações, em outubro de 1958 Paul DeHart Hurd publicou um artigo no qual o termo scientific literacy foi utilizado como sendo um dos objetivos da educação científica (DEBOER, 2000; HOLDBROOK, RANNIKMAE, 2009; TORRES, ZAPATA, PINTO, 2009; BYBEE, 2012). Neste momento histórico, a comunidade estadunidense deparava-se com a Guerra Fria (1945-1991) marcada, dentre outros fatos, pelo lançamento, em 4 de outubro de 1957, do satélite Sputinik pela já extinta União Soviética, o que gerou a necessidade de implementar o desenvolvimento científico, por meio do ensino de Ciências, em prol desta "corrida aeroespacial". Porém, Hurd preocupou- 
se que os objetivos de ensinar ciências para fomentar a autonomia e liberdade fossem sobrepostos pela necessidade momentânea de desenvolvimento tecnológico "Serão os pesadores sobre currículo capazes de desenvolver um programa educacional que possa manter o delicado balanço das forças cientificas, sociais e econômicas que serão encontradas neste período?" (HURD, 1958, p. 14, tradução nossa).

Para Robert (1983), o período entre 1950 e 1970 legitimou a terminologia, uma vez que começaram a surgir várias definições e conceitos para o termo o que caracterizavam seu dinamismo. Para DeBoer (2000), neste período o que ocorreu na prática foi a supremacia do desenvolvimento de habilidades práticas imediatas em detrimento do conceito integrador que abarca as relações da ciência a sociedade.

Na década de 1970 a National Science Teachers Association (NSTA) redefine o termo, retomando a relação da ciência e sociedade, colocando a alfabetização científica como sendo a utilização de conceitos, habilidades e valores científicos na tomada de decisões diárias, na interação com outras pessoas e com o meio ambiente, de forma a compreender a inter-relações entre a ciência, a tecnologia, a sociedade e a economia consolidando a terminologia no contexto social (DEBOER, 2000).

O contexto histórico americano da década de 1970, marcada pelo afloramento da economia do Japão que levou ao declínio da supremacia industrial e econômica americana, que somada a decadência das pesquisas em ciências e engenharia em comparação ao cenário mundial corroboraram para reforçar a correlação da ciência e da tecnologia como conhecimentos básicos para o desenvolvimento do pais, o que justifica o enfoque dado pela NSTA (LAUGKSCH, 1999).

Nesse período emerge o currículo com enfoque CTS - Ciência, Tecnologia, Sociedade e Ambiente que objetiva evidenciar e discutir as relações entre a ciência, a tecnologia a sociedade e o ambiente fomentando a cidadania. Todavia, esta proposta encontrou opiniões contrárias que se pautavam no empobrecimento da aprendizagem dos conceitos básicos ao afirmar que, nesta situação, os conhecimentos essenciais não são ensinados, justificando este fato pela característica transitória dos contextos sociais (DEBOER, 2000).

Para Laugksh (1999), a década de 1980, pautada por uma crise industrial, econômica, científica e educacional, serviu como forma motriz para o desenvolvimento da terminologia. O autor relata que, no período de 1970 a 1990, foram publicados mais de 330 artigos americanos sobre alfabetização científica, sendo a maioria posterior a 1980.

DeBoer (2000) narra que em 1983 um relatório produzido pela National Commission on Excellence in Education intitulado "A Nation at Risk: The Imperative for Educational Reform" revelava evidencias de empobrecimento dos padrões educacionais, caracterizado pelo baixo rendimento dos estudantes em avaliações, especialmente em Ciências, o que indicava a necessidade de ressignificar os processos de ensino e aprendizagem. Neste panorama, a solução foi modificar os currículos escolares, tornando-os mais exigentes quanto ao ensino de Inglês, Matemática, Ciências Naturais e Ciências Humanas, Informática e Línguas Estrangeiras. Estas modificações tinham como objetivo, melhorar o rendimento escolar dos alunos com consequente potencialização do desenvolvimento econômico e industrial americano, recolocando os EUA no cenário mundial.

A situação descrita ocasionou dois movimentos de scientific literacy, um baseado na aprendizagem, com rigor, de conceitos e conhecimentos, e outro baseado na proposta CTS (OSBORNE, 2007). No entanto, mesmo após as modificações curriculares e, por consequência, metodológicas, acima indicadas, o desenvolvimento da nação americana não 
respondeu rapidamente, em comparação a outros pais, fato que levou a novo movimento nas reformas educacionais.

Surge a AASS - American Association for Adevancement of Science - que propõe o slogan "Science for all American" por meio do projeto 2061 que resgata a relação CTS na terminologia alfabetização científica.

[...] sendo familiar com o mundo natural e respeitando sua unidade, estando atento aos importantes caminhos nos quais as ciências exatas, a tecnologia e as ciências gerais dependem umas das outras, entendendo alguns dos conceitos chave e princípios da ciência, tendo a capacidade de elaborar o pensamento científico, sabendo que as ciências exatas e tecnologia são empreendimentos humanos e sabendo o que implica em suas forças e limitações, e sendo capaz de usar o conhecimento cientifico, modos de pensar para propósitos pessoais e sociais (AAAS, n.p, 1989, tradução nossa).

A proposta do projeto 2061 motivou a Nacional Academy of Sciency por meio da publicação do Nacional Educational Standarts em 1996, a estabelecer parâmetros necessários para que todos os estudantes pudessem ser cientificamente alfabetizados, sendo estes: (a) todos precisam fazer uso do conhecimento cientifico para a tomada de decisões diárias; (b) todos precisam ser capazes de se envolver ativamente em discussões que envolvam questões científicas e tecnológicas; (c) todos devem compartilhar da satisfação pessoal provinda da compreensão do mundo natural; (d) todos devem ser capazes de aprender, pensar e pensar criativamente na buscar por soluções de problemas por meio da compreensão dos processos científicos; (e) todos devem ter a possibilidade de protagonizar a atuação cidadã. (DEBOER, 2000, p.590 - tradução do autor). Desta forma, de acordo esses novos padrões, uma pessoa alfabetizada cientificamente

Significa que uma pessoa que tem a habilidade de descrever, explicar e prever fenômenos naturais. A Alfabetização Científica implica em ser capaz de ler e interpretar artigos sobre ciência em mídias populares e se envolver em conversas sociais sobre a validade destas conclusões. Alfabetização Científica implica que uma pessoa pode identificar questões científicas subjacentes a decisões nacionais e locais e expressar posições que são cientificamente e tecnologicamente válidas, um cidadão alfabetizado cientificamente deve ser capaz de avaliar a qualidade da informação científica desde sua fonte e dos métodos usados para gerá-la. A educação científica também implica na capacidade de apresentar e validar argumentos baseados em evidência e aplicar conclusões sobre tais argumentos de forma apropriada (NATIONAL SCIENCE EDUCATION STANDARDS In: DEBOER, 2000, P.591, tradução nossa).

Desta forma, fica evidente que uma pessoa cientificamente alfabetizada não apenas é capaz de entender os conhecimentos, ideias, propostas e conceitos científicos como os reconhece e aplica em situações da vida diária, tanto no âmbito individual (decisões da vida pessoa), como no âmbito coletivo (decisões enquanto cidadão), sendo capaz de compreender e interpretar o mundo em que vive, com autonomia necessária, tanto para o ativismo cidadão, quanto para a satisfação pessoal, o que torna o exercício de educar para a alfabetização científica uma tarefa altamente complexa. 
Nesta perspectiva, o PISA - Programme for International Student Assessment - de 2015 define o letramento cientifico como sendo as capacidades individuais de: (a) explicar fenômenos cientificamente, o que exige dos alunos conhecer os conceitos científicos, ou seja dominar os conhecimentos conceituais, que são aqueles construídos pela comunidade científica, tal como definido por Zabala (1998); (b) avaliar e planejar experimentos científicos, essenciais para que o alunado compreenda a natureza da ciências, como as investigações científicas são propostas, planejadas e conduzidas reconhecendo quais são as características de uma investigação científica o que podemos entender como sendo o conhecimento procedimental proposta por Zabala (1998); (c) interpretar dados e evidencias científicas, o que exige apoderar-se da epistemologia da ciência para compreender hipóteses, perguntas, questionamentos, averiguações, teorias, modelos e argumentos na ciência, reconhecendo a importância das investigações científicas e das situações alvo destas. Além disso os autores do PISA destacam que o uso do termo Letramento Científico oferece a possibilidade que estas competências sejam aplicadas no dia a dia, ou melhor, no contexto das situações de vida dos educandos.

Entretanto, as competências alvos de investigação do PISA não abarcam a relação CTS que fomentou a inserção da alfabetização científica como alvo das reformas mundiais no ensino de Ciências. Para lan Milner (2004), a alfabetização científica objetiva, não somente a aquisição de conhecimentos acerca da ciência e da tecnologia, no tocante dos conceitos, procedimentos e epistemologia, mas também das aplicações e tomada de decisões diárias. Para o autor a capacidade de tomar decisões acerca do meio ambiente, da saúde e do bem-estar, tendo como base a ciência, também é importante, pois,

A educação científica nas escolas deve focar no desenvolvimento do conhecimento infantil, habilidades, atitudes e valores que irão permiti-los tomar uma posição baseada em questões científicas que venham a enfrentar em sua vida cotidiana. Os formandos devem estar cientes de e ter um entendimento do processo cientifico e dos seus valores sociais (MILNER, 2004, p.2, tradução nossa).

De acordo com Graça (2009) e Pella e colaboradores (1966), em uma meta análise de mais de 100 artigos, os pesquisadores puderam identificar seis dimensões que uma pessoa cientificamente alfabetizada devem possuir: i) conceitos básicos de ciências; ii) a natureza das ciências; iii) a ética que controla dos trabalhos da ciência; iv) as inter-relações da ciência com a sociedade; v) as inter-relações da ciência com a humanidade vi) as diferenças entre ciência e a tecnologia.

Ainda, Showalter (1974) delineou sete dimensões de uma pessoa cientificamente alfabetizada: i) a compreensão da natureza dos conhecimentos científicos; ii) a aplicação apropriada dos conceitos, princípios, leis e teorias da ciência na interação com o universo; iii) a utilização dos processos da ciência na resolução de problemas para a tomada de decisão e para a sua própria compreensão do universo; iv) a interação com os vários aspectos do universo de forma consistente com os valores subjacentes à ciência; v) a compreensão das relações entre a ciência e a tecnologia e as inter-relações de cada uma destas com a sociedade; vi) o desenvolvimento de uma visão holística e científica do universo, sendo este estimulante para perpetuar a cultura científica; vii) o desenvolvimento de habilidades práticas acerca da ciência e a tecnologia.

Também, Sasseron e Carvalho (2008) apresentam, além da evidente importância da aprendizagem de conceitos, procedimentos e aspectos epistemológicos, a valorização da 
aprendizagem dos conhecimentos atitudinais, ou seja, aqueles relacionados com os valores, reflexões e ações articuladas pelos educandos.

Corroborando essas ideias, Laugksch (1999) expõe que a perspectiva de alfabetização científica adotada depende do grupo de interesse direcionado, ou seja, quando há o interesse dos educadores em ciências, que objetivam a reforma educacional, as discussões sobre alfabetização científica rumam para os objetivos da educação científica, como a formação de valores, atitudes e habilidades implica no currículo de ciências, como atingir os objetivos da educação científica com eficiência, e como avalia-los. Assim, quando a comunidade escolar tem como foco a alfabetização científica, as suas dimensões expressam as relações entre ela e a educação formal.

Já, quando o foco são pesquisadores da área da divulgação científica, a alfabetização científica expressa, de forma mais enfática, as relações entre a ciência e tecnologia na sociedade, ou seja, a possibilidade da participação publica nas decisões políticas, econômicas, sociais, científicas e ambientais tendo a ciência e suas possibilidades como norteadoras (LAUGKSCH, 1999).

O terceiro grupo de interesse são aqueles que discutem a alfabetização científica segundo uma perspectiva sociológica, portanto, os profissionais da área das Ciências Sociais. Para este grupo a autoridade científica, bem como os contextos de propagação e divulgação das ideias são fundamentais. Desta forma, como a sociedade tem acesso aos conhecimentos científicos e como este é interpretado de forma que ocorra a distinção entre opiniões com base em aspectos científicos de opiniões isoladas, ganham uma proporção mais significante (LAUGKSCH, 1999)

Por fim, ainda para o mesmo autor, o último grupo de interesse é classificado como profissionais que atuam na educação não formal, ou seja, museus, exposições cientificais, sites e eventos que possam promover o acesso o conhecimento científico fora do ambiente escolar. Para este grupo o interesse seria em promover a alfabetização científica, principalmente para crianças, adolescentes e adultos de forma lúdica, sendo utilizadas diferentes abordagens em espaços dinâmicos, para públicos diversos. Assim, é compreensível que cada situação de interesse, a perspectiva de alfabetização científica se aproprie de tendências e significados, adquirindo fundamentos, eixos e definições distintas.

Porém, para Shen (1975) não existe apenas um tipo de alfabetização científica, sendo esta aplicada a diferentes necessidades, situações e populações. Desta forma, o autor classificou a alfabetização científica em três tipos: i) alfabetização científica prática que contempla a aquisição de conhecimentos para a solução de problemas do dia a dia, como fazer uso de um dado medicamento ; ii) alfabetização científica cívica, na qual os conhecimentos científicos sãos aplicados aos princípios de cidadania e democracia; iii) alfabetização científica cultural, na qual os conhecimentos são percebidos como constructos humanos, integrando a cultura científica, evidenciando que a alfabetização científica pode existir em diferentes situações não excludentes.

Para Gabel (1976) a alfabetização científica apresenta-se em níveis. O autor, tendo como base os trabalhos de Bloom - a Taxonomia de Bloom - descreve a alfabetização científica tendo como base o desenvolvimento de habilidades e capacidades cognitivas que parte da identificação de conhecimentos - nível 1 - até a avaliação - nível 6.

Ainda, para Shamos (1995), a alfabetização científica também se apresenta em níveis sendo esses: (i) alfabetização científica cultural, na qual o conhecimento é utilizado na comunicação básica; (ii) alfabetização científica funcional, na qual os indivíduos são capazes 
de ler, escrever e conversar utilizando termos científicos em situações informais, como consultas médicas, blogs, sites de comunicação e marketing como Facebook e Youtube e a (iii) alfabetização científica verdadeira, por meio da qual o indivíduo entende a natureza da ciência bem como as investigações científicas são conduzidas e relacionadas.

Nesta mesma perspectiva, Bybee (1997) propõe que a alfabetização científica ocorre de forma processual e gradual partindo de um (i) nível nominal, no qual a indivíduo reconhece termos científicos, para um (ii) nível funcional, que prevê a utilização do vocabulário científico e tecnológico, porém em situações descontextualizadas, como em uma prova escolar, tendo a possibilidade de evoluir para um (iii) nível conceitual e procedimental no qual, além de fazer uso do vocabulário científico, é capaz de entender e demonstra-los, culminado no (iv) nível multidimensional, no qual o indivíduo, compreendendo a natureza da ciência e suas relações com a tecnologia e sociedade, fazer uso destes conhecimento na sua vida pessoal e social.

Afastando-se desta proposta de entender a alfabetização científica em níveis, Sasseron e Carvalho (2008), após realizarem uma extensa revisão bibliográfica sobre a alfabetização científica, destacam que é possível encontrar convergência entre os autores sendo possível a compilação destas ideias em três eixos que podem ser foco de reflexão docente no processo de planejamento do ensino e aprendizagem em ciências: (i) compreensão básica de termos, conhecimentos e conceitos científicos fundamentais no qual os cabe aos alunos conhecer e compreender os conceitos e ideias fundamentais da ciência, na perspectiva de aplica-los em situações do dia a dia; (ii) compreensão da natureza das ciências e dos fatores éticos e políticos que circundam sua prática, ou seja, a compreensão da ciência como um corpo de conhecimentos dinâmico, resultante do processo de aquisição e análise de dados que sintetizam e decodificam resultados que culminam na construção do conhecimento; (iii) entendimento das relações existentes entre ciência, tecnologia, sociedade e meio-ambiente que trata da inter-relação entre as dimensões acima citadas.

A proposta das professoras Sasseron e Carvalho (2008), alicerçada nas ideias de Paulo Freire acerca da alfabetização, foi aplicada nos anos iniciais do ensino fundamental com o objetivo de entender como as propostas de sala de aula permitem determinadas ações e atitudes de alunos e professor de modo que estes elementos se relacionam e propiciam discussões e debates acerca das ciências, suas tecnologias e os impactos destas duas para a sociedade e o ambiente. Para tanto, as autoras partem da compreensão de que a alfabetização não será atingida no ensino fundamental, mas, uma vez iniciada, deverá estar em constante construção, o que evidencia o caráter processual da alfabetização científica na compreensão das mesmas.

As autoras destacam, ainda, que conforme novos conhecimentos são introduzidos, serão desenvolvidas habilidades que evidenciam o processo de alfabetização científica, sendo estes os indicadores de alfabetização científica (IAC):

i) Indicadores derivados do trabalho com dados empíricos:

i.i) Seriação da informação: remete a seleção de informação para a ação investigativa;

i.ii) Organização da informação: emerge quando os dados são preparados para que o processo investigativo possa acontecer;

i.iii) Classificação da informação: aparece quando se busca estabelecer características para os dados obtidos no processo investigativo;

ii) Indicadores derivados da construção de argumentos nas aulas de ciências: 
ii.i) Raciocínio lógico: compreende o modo como as ideias são desenvolvidas e apresentadas;

ii.ii) Raciocínio proporcional: além de apresentar a organização do pensamento revela como as variáveis se inter-relacionam;

iii) Indicadores de levantamento de hipóteses: Levantamento de suposições acerca de um tema;

iv) Indicadores de testes de hipóteses: Caracteriza-se pelas etapas em que o indicador anterior é colocado a prova;

v) Indicadores de justificativa: caracteriza-se pelo aparecimento de fatores que possam avalizar, dar garantias acerca das ideias propostas;

vi) Indicadores de previsão: Aparece quando uma ação ou fenômeno está associado a eventos que se sucedem;

vii) Indicativo de explicação: Aparece quando se buscar relacionar informações a hipóteses já levantadas

Estes indicadores de alfabetização científica podem apontar a possibilidade de que os alunos estejam, de fato, relacionando ideias e conceitos as perspectiva epistemológicas, éticas, procedimentais, e atitudinais no qual a proposta de se trabalhar alfabetização científica como promotora da educação científica na educação básica emerge. Assim, diante desta emergência do ensino de Ciências, outras áreas do conhecimento, também alicerçadas na necessidade de preparar o cidadão para o mundo atual, buscaram na alfabetização científica fundamentos, eixos e indicadores que pudessem fomentar esta proposta, dentre elas a saúde e a nutrição e alimentação humana.

\section{A alfabetização em saúde e nutrição humana}

O conceito de alfabetização, para além do desenvolvimento das habilidades de leitura e escrita, deve promover a comunicação por meio da inter-relação entre a cultura e a sociedade, ou seja, um indivíduo alfabetizado está integrado a realidade, pois é capaz de realizar uma leitura crítica, expondo suas opiniões e ideias.

Partindo dessa premissa, Kickbusch (2001) apresentou algumas modalidades de alfabetização que uma pessoa precisa dominar para viver na sociedade atual, intitulando-as: alfabetização qualitativa, alfabetização científica, alfabetização tecnológica, alfabetização cultural, alfabetização midiática, e alfabetização em computação. Para o mesmo autor, a alfabetização em saúde deveria integrar esta lista.

Porém, a alfabetização em saúde já vem sendo discutida desde a década 1970, sendo inicialmente descrita como a capacidade do paciente de seguir as prescrições médicas acerca da terapêutica recomendada (AD HOC COMMITTEE ON HEALTH LITERACY FOR THE COUNCIL ON SCIENTIFIC AFFAIRS, AMERICAN MEDICAL ASSOCIATION, 1999).

Atualmente, esta é definida pelo CDC - Centers of Desease Control and Prevention (2017, p.1) - como "a capacidade individual de obter, comunicar e processar conhecimentos básicos de saúde bem como tomar decisões apropriadas quanto a própria saúde.

Para o Institut of Medicine (2004), um cidadão alfabetizado em saúde é capaz de dominar os conhecimentos básicos sobre saúde, bem como a cultura local, podendo falar, ler, escrever e entender as perspectivas numéricas a respeito da saúde humana. Todavia, para que um cidadão possa desenvolver essas capacidades, se faz necessário a compreensão de como o organismo humano funciona, como e por quais razões o corpo 
adoece, compreender quando e onde buscar informações sobre a saúde, avaliar se as informações encontradas são confiáveis, identificar e compreender os sintomas bem como os tratamento e ações preventivas e profiláticas (ZARCADOOLAS et al., 2006; RUDD, ANDERSON, NATH, 2007).

Ainda, de acordo com Nutbeam (2008) a alfabetização em saúde, assim como a alfabetização científica, encontra múltiplas definições podendo ser classificada em (a) um conjunto de habilidades individuais ou (b) como interação prática.

A alfabetização em saúde como um conjunto de habilidades individuais é definida como o grau em que o indivíduo obtém, processa e compreende, informações básicas de saúde de forma a tomar decisões conscientes acerca de sua saúde (DEPARTMENT OF HEALTH AND HUMAN SERVICES, 2000). Porém, esta proposta isenta o indivíduo de analisar como o sistema de saúde é organizado bem como quais são as políticas públicas executadas, qual é qualidade do material de divulgação científica e educação em saúde produzidos, ou seja, esta percepção de alfabetização em saúde pode ser considerada higienista ou sanitarista, pois objetiva a melhora da qualidade de vida do sujeito sem que ocorra a reflexão sobre a saúde na magnitude cidadã.

Já a alfabetização em saúde como uma integração prática considera tanto o indivíduo a ser alfabetizado quanto os profissionais e instituições envolvidos no processo de alfabetização, sendo "a alfabetização em saúde um estado dinâmico que pode depender de uma variedade de fatores, incluindo o problema médico a ser tratado, o provedor de cuidados de saúde e o sistema que presta cuidados (INTITUTE OF MEDECINE, 2004, p.2 2006, tradução nossa). Para Edward at al, (2012), essa perspectiva tem uma compreensão dinâmica e processual da alfabetização em saúde pois, a considera como uma construção multidimensional que ocorre em diferentes contextos e interações.

Nesta perpspectiva, Leger (2001) apresenta três níveis de alfabetização em saúde: (a) funcional, que compreende a capacidade de aprender, comunicar e informar sobre: higiene, nutrição, segurança, drogas, relacionamento, sexualidade, paternidade/maternidade; (b) interativa, que abarca o desenvolvimento de habilidade pessoais como resolver problemas, preparar alimentos, os cuidados com a higiene pessoal e a comunicação utilizando a linguagem científica apropriada; (c) crítica, que objetiva a participação dos sujeitos em iniciativas sociais que possibilitem o desenvolvimento e efetivação de políticas públicas de saúde bem como ações para fomentar a qualidade de vida da sociedade.

Este roll de capacidades necessárias para que a alfabetização em saúde ocorra está intimamente relacionado com o sistema de saúde existente, ou seja, com as dimensões políticas, econômicas e ambientais existentes, a sociedade local e com os conhecimentos básicos sobre saúde que são fornecidos para a população, incluindo os conhecimentos escolares (BACKER, 2006).

A escola configura-se como um espaço privilegiado para a construção de conceitos e conhecimentos sobre saúde e o desenvolvimento de hábitos saudáveis, sendo considerada por Leger (2001) como "primary establishment" na construção de capital e saúde de uma nação. Para Denuwara e Gunawardena (2017) o nível crítico da alfabetização em saúde será atingido mais rapidamente se a escola básica (compreendida pelo ensino fundamental e médio) assumir a função de promoção da alfabetização em saúde.

De acordo com Père-Rodrigo e Aranceta (2003), na idade escolar, os alunos começam a tomar as próprias decisões no que tange o desenvolvimento de hábitos saudáveis ou não saudáveis. Nesse processo, a família passa a ter menos importância, enquanto os amigos, 
bem como os grupos sociais e as tendências midiáticas acabam por serem fatores decisivos, desta forma, a escola tem um papel determinante, sendo a instituição que mais impacta na promoção da saúde, e, portanto, na alfabetização em saúde (ALDINGER, JONES,1998; DIXEY et al, 1999). Assim, a escola, na condição de promotora da saúde, deveria considerar os interesses dos alunos, dos professores e da própria escola em si, sendo, todos atores fundamentais e ativos nesse processo.

\section{A alfabetização em nutrição e alimentação}

A alfabetização em nutrição ou alfabetização em alimentação compreende a alfabetização em saúde segundo um contexto nutricional.

Para Pettersen et al. (2009), Silk et al. (2008) e Velardo (2015), a alfabetização em nutrição e alimentação deriva da alfabetização em saúde multidimensional, é compreendida em três níveis:

(a) alfabetização nutricional funcional, na qual o indivíduo deve ser capaz de obter e compreender informações básicas sobre nutrição como forma de melhorar sua qualidade de vida, tais como: os componentes de uma alimentação saudável, morbidades e riscos para a saúde humana e os benefícios da alimentação saudável, ou seja, o que os autores chamam de conhecimento declarativo ou processual. O conhecimento declarado é caracterizado pela ciência dos fatos e processos da área de conhecimento a ser estudada. Já o conhecimento processual relaciona-se ao desenvolvimento de habilidades, competências e estratégias acerca de como executar algo, ou seja, o caráter prático do conhecimento.

(b) alfabetização nutricional interativa, que objetiva o desenvolvimento de habilidades cognitivas e interpessoais para gerenciar situações em parceria com profissionais da área de nutrição. Nesta perspectiva, o conhecimento nutricional é entendido como integrante de um contexto. Para os autores que defendem essa proposta, os conhecimentos nutricionais adquiridos precisam ser aplicados em situações cotidianas, como o preparo da refeição, a compra de alimentos, ou seja, as escolhas alimentares. Para Zoellner et al (2009), um cidadão que se encontra neste nível de alfabetização em nutrição é capaz de utilizar os guias alimentares - guidelines - bem como as tabelas nutricionais em situações práticas.

(c) Alfabetização nutricional crítica, esta deve permitir que o cidadão analise criticamente situações, promovendo a conscientização e efetivação de ações de enfrentamentos de problemáticas que envolvem a alimentação e a nutrição humana, tais como: o uso de substância químicas na produção de alimentos, o posicionamento político e ambiental das empresas produtoras de alimentos, a qualidade da merenda escolar, a utilização de alimentos em propagandas, ou seja, cabe ao indivíduo perceber os problemas sociais, econômicos, ambientais e científicos que permeiam a alimentação humana propondo ações de enfrentamento e analisando propostas de forma a ter uma atuação cidadã.

Ainda, de acordo com Vidgen e Gallegos (2014), o desenvolvimento de habilidades relacionadas a gastronomia e culinária, tais como, o preparo de refeições e pratos, técnicas de cocção, técnica de medição de ingredientes, técnicas de congelamento e descongelamento, devem ser consideradas como importantes na alfabetização em nutrição. Para alguns autores - a alfabetização em nutrição que objetiva o desenvolvimento de 
habilidades culinárias pode ser identificada como alfabetização em alimentos - food literacy (VIDGEN, 2016).

\section{A correlação entre a alfabetização científica, alfabetização em saúde e alfabetização em nutrição: suas inter-relações, possibilidades e limitações para o ensino de Ciências}

A alfabetização, compreendida como um processo emancipatório que, além de fornecer subsídios para a aprendizagem das linguagens, ou seja, compreensão da simbologia que envolve a comunicação, é capaz de promover a formação cidadã, fornecendo subsídios para que o sujeito em formação possa conhecer o mundo em que vive, identificando os fatores sociais, éticos, políticos, econômicos, ambientais e científicos bem como suas inter-relações.

Nesta perspectiva, a alfabetização em saúde objetiva integrar as possibilidades acima listadas como parte do processo de promoção da saúde. Processo este que deixa de ter seu foco apenas no estado de saúde/doença, que é pautado em uma perspectiva higienista, para admitir que um indivíduo que não é capaz de entender as Ciências da Saúde, minimamente em um nível básico, terá menos chance de alcançar qualidade de vida.

Ainda, a nutrição, como integrante das Ciências da Saúde, tem percebido e correlacionado estatisticamente que sujeitos que detém pouco conhecimento sobre nutrição, bem como, habilidades básicas acerca da área de conhecimento, também tem chances menores de desenvolver hábitos alimentares saudáveis contribuindo para a boa saúde.

Diante disso, é possível correlacionar as três tendências de forma a obter pontos de convergência, conforme o proposto na Quadro 1.

\begin{tabular}{|c|c|c|c|}
\hline & Alfabetização científica & Alfabetização em Saúde & Alfabetização em Nutrição \\
\hline$\hat{\bar{\nu}}$ & $\begin{array}{l}\text { 1.Compreensão básica } \\
\text { de termos, } \\
\text { conhecimentos e } \\
\text { conceitos científicos } \\
\text { fundamentais; } \\
\text { 2.Compreensão da } \\
\text { natureza das ciências e } \\
\text { dos fatores éticos e } \\
\text { políticos que circundam } \\
\text { sua prática; } \\
\text { 3.Entendimento das } \\
\text { relações existentes entre } \\
\text { ciência, tecnologia, } \\
\text { sociedade e meio- } \\
\text { ambiente; }\end{array}$ & $\begin{array}{l}\text { 1. Funcional: capacidade de } \\
\text { aprender, comunicar e informar } \\
\text { sobre: higiene, nutrição, segurança, } \\
\text { drogas, relacionamento, } \\
\text { sexualidade, } \\
\text { paternidade/maternidade; } \\
\text { 2.Interativa: compreende o } \\
\text { desenvolvimento de habilidade } \\
\text { pessoais: resolver problemas, } \\
\text { preparar alimentos, higiene e } \\
\text { comunicação; } \\
\text { 3. Crítica: participação de iniciativas } \\
\text { sociais que possibilitem o } \\
\text { desenvolvimento e efetivação de } \\
\text { políticas públicas de saúde. }\end{array}$ & $\begin{array}{l}\text { 1.Funcional: integra habilidades de } \\
\text { leitura e escrita necessárias para a } \\
\text { compreensão dos conceitos } \\
\text { básicos de nutrição; } \\
\text { 2. Interativa: habilidades cognitivas } \\
\text { e interpessoais para gerenciar } \\
\text { situações em parceria com } \\
\text { profissionais da área de nutrição; } \\
\text { 3.Crítica: analisar criticamente } \\
\text { situações, promovendo a } \\
\text { conscientização e efetivação de } \\
\text { ações de enfrentamentos de } \\
\text { problemáticas que envolvem a } \\
\text { alimentação e a nutrição humana; }\end{array}$ \\
\hline
\end{tabular}

Quadro 1. Perspectivas e tendências acerca Alfabetização Científica, Alfabetização em Saúde e Alfabetização em Nutrição. 
Na tabela acima apresentada fica evidente que para as três propostas, a primeira possibilidade a ser almejada é a identificação e compreensão dos conceitos básicos da ciência em questão, ou seja, tomar posse da produção científica básica até o momento disponível. Diante disso, podemos afirmar que é de extrema importância que, no que tange os conteúdos de alimentação e nutrição, os conhecimentos básicos desta ciência sejam tratados no processo de escolarização.

Desta forma, é imprescindivel estabelecer quais conceitos, teorias e paradigmas da nutrição devem ser tratados na educação básica, pois estes são essenciais para que a alfabetização em nutrição possa ser precoce e mais efetiva.

$\mathrm{Na}$ tentativa de avaliar o nível de alfabetização em nutrição, Diamond (2007) desenvolveu uma escala, inicialmente validado para adultos, mas que posteriormente foi validado por Leslee A. D'amato-kubiet (2013) para ser aplicado em adolescentes. Nesta escala, o autor, por meio de questões de múltipla escolha, que são conduzidas como opções para completar um texto, considera a compreensão de conceitos como, a função da alimentação saudável, composição nutricional dos alimentos, ingestão calórica, ingestão de nutrientes, o consumo de gorduras, os tipos de gorduras existentes, o consumo de fibras e grão integrais, o consumo de cálcio, de vitamina D, os conceitos de "calorias vazias" , higiene e armazenamento dos alimentos, alimentos orgânicos e a relação da alimentação com o aumento de peso.

Ainda nesta perspectiva, Appleton (2010) validou a aplicação, em adolescentes, do Neweste Vital Sign Health Literacy Assessment que avalia o nível de Alfabetização em Saúde com base na leitura e interpretação do rótulo de um sorvete. Neste texto são aplicadas seis perguntas que tem como foco avaliar o tamanho das porções, a composição dos alimentos e as alergias alimentares.

Ainda nesta perspectiva, Gibbs (2012) em sua tese se doutorado desenvolveu múltiplos instrumentos para avaliar a alfabetização em nutrição tendo como foco da pesquisa a determinação do tamanho das porções alimentares, a composição dos alimentos, a proporção dos macronutrientes e micronutrientes presentes em alimentos de diferentes grupos alimentares, a habilidade de interpretação de rótulos de alimentos e a classificação dos alimentos em grupos.

No Brasil, as pesquisas que buscaram identificar a alfabetização em nutrição são escassas, porém, Sampaio et al. (2013) em um estudo amplo, realizado de 2009 a 2011, como parte do Plano AlfaNutri: um novo paradigma, a alfabetização nutricional, para promoção da alimentação saudável e prática regular de atividade física na prevenção e controle de doenças crônicas', financiada pelo Ministério da Saúde/Conselho Nacional de Desenvolvimento Científico, no qual a alfabetização em nutrição de 2 grupos, jovens adultos pacientes do SUS e jovens adultos frequentadores se shopping centers foi avaliada por meio do Nutritional Literacy Scale - NLS (Diamond, 2007), o que evidencia a falta de instrumentos validados desenvolvidos para a população brasileira, sendo esta uma das indicações dos autores.

Os mesmos autores, em 2014, realizaram um outro estudo comparando a aplicação de dois métodos de análise acerca da alfabetização em nutrição na população brasileira, o Nutritional Literacy Scale - NLS (Diamond, 2007) e o Neweste Vital Sign Health Literacy Assessment (Apleton, 2010), revelando que os métodos não são intercambiáveis e que houve uma diferença significativa no resultado dos dois para a mesma população, ou seja, 
ambos os métodos podem não ser adequados para a avaliação da Alfabetização em Nutrição na população brasileira. Não foram encontrados outros estudos no Brasil.

Diante da situação acima exposta, é evidente que não existe, ainda, um investimento nacional na alfabetização em nutrição, o que reflete nos possíveis enfrentamentos acerca desta problemática na educação básica. Ademais, Bizzo e Leder (2005) apontam que a educação nutricional trabalhada nas escolas ocorre sempre com um caráter higienista, ou seja, como uma simples verificação de conhecimentos. As autoras destacam a alfabetização em saúde e a alfabetização em nutrição como forma de empoderamento, pois instrumentalizam o cidadão na obtenção da qualidade de vida, enfatizando que este deveria ser o objetivo da educação nutricional na escola e no currículo escolar.

Partindo desta premissa, e tendo como base os estudos analisados neste trabalho, apontamos quais conteúdos precisam ser trabalhados na educação básica para a promoção da alfabetização em nutrição partindo da perspectiva da alfabetização científica:

- Alfabetização Nutricional Funcional: é aquela na qual os alunos identificam e compreendem os conceitos básicos da nutrição, tendo em vista a cultura, a economia, o meio ambiente a sociedade local, sendo estes: bioquímica e tecnologia dos alimentos, princípios fundamentais da nutrição humana e a relação da alimentação com o ser humano, enfatizando tanto o binômio saúde/doença quanto as questões culturais e religiões

- Alfabetização Nutricional Crítica: é aquela na qual o indivíduo é capaz de aplicar os conteúdos e conhecimentos básicos de nutrição em situações reais, ou seja, diferentes contextos, relacionando-os aos diversos domínios e dimensões humanas tais como a política, a economia, a sociedade, o meio ambiente, a cultura e a ciência de forma a promover a participação pública cidadã bem como o enfrentamento de problemáticas da atualidade. Assim, a escola deve promover o processo de ensino e aprendizagem de forma a fomentar a inter-relação entre as dimensões, bem como situações problemas nas quais os estudantes possam fazer uso dos conhecimentos nutricionais na análise crítica de situações, refletindo e propondo ações. Um exemplo disso seria discutir a qualidade da merenda, a produção de alimentos na região, o preço dos alimentos e sua relação com a política local, a ditadura da magreza, os transtornos alimentares, as dietas da moda, dentre outras situações com potencial promotor. O diferencial desta modalidade é que, além de identificar e compreender conceitos, espera-se que o aluno desenvolva ações pautadas dos indicadores de alfabetização científica

\section{Considerações finais}

A alfabetização em nutrição, que é compreendida dentro da alfabetização em saúde, que por sua vez está contida na alfabetização científica, enquanto possibilidade para a educação científica básica (composta pela educação infantil, ensino fundamental e médio), busca promover, não somente a memorização de conceitos específicos da ciência da nutrição como também uma reflexão epistemológica que pode promover o desenvolvimento de habilidades específicas capazes de possibilitar o desenvolvimento da autonomia quanto ao gerenciamento consciente de seus hábitos alimentares e de vida além da atuação cidadã na proposta, análise e fiscalização de políticas públicas quanto a temática em questão o que pode influenciar, significativamente, da melhora da qualidade de vida refletindo na saúde pública como um todo. 


\section{Referências}

AD HOC COMMITTEE ON HEALTH LITERACY FOR THE COUNCIL ON SCIENTIFIC AFFAIRS, AMERICAN MEDICAL ASSOCIATION. Health Literacy Report of the Council on Scientific Affairs. JAMA, February 10, v, 281, n. 6, 1999.

ALDINGER, C.E.; JONES, J.T. Healthy Nutrition: An Essential Element of a Health-promoting School. WHO. Information Series on School Health. Document four. Geneva: WHO, 1998.

AMERICAN ASSOCIATION FOR THE ADVANCEMENT OF SCIENCE - AAAS. (1989). Science for all Americans. New York: Oxford University Press. n.p. Disponível em <http://www.project2061.org/publications/sfaa/online/sfaatoc.htm.>. Aces. jul. 2017.

APLETON, A. A. Promoting health literacy through the school nutrition environment. Graduate theses and Dissertation. lowa State Universaty, 2010. Disponível em $<$ http://lib.dr.iastate.edu/cgi/viewcontent.cgi?article=2195\&context=etd $>$. Acesso em Jun, 2017.

AULER, D.; DELIZOICOV, D. Ciência-Tecnologia-Sociedade: relações estabelecidas por professores de Ciências. Enseñanza de las Ciencias, v. 5; n.2, p. 337-355, 2006.

AULER, D.; DELIZOICOV, D. Alfabetização científico-tecnológica para quê? Ensaio -Pesquisa em Educação em Ciências, v. 3, n. 1, p. 1-13, 2001.

BACKER, D. W. The meaning and measure of health literacy. Journal of General Internal Medicine, n.21, p.878-883, 2006.

BIZZO, M.L.C; LEDER, L. Educação nutricional nos parâmetros curriculares nacionais para o ensino fundamental. Campinas: Rev. Nutr, v.18, n.5, p.661-667, 2005.

BRANDI, A. T. E.; GURGEL, C. M. A. A alfabetização científica e o processo de ler e escrever em séries iniciais: emergências de um estudo de investigação-ação. Ciência \& Educação, Brasília, v. 8, n. 1, p.113-125, 2002.

BYBEE, W. R. Scientific Literacy in Environmental and Health Education. Science Environmental Health, 2012.

CARVALHO, A.M.P; TINOCO, S. C. O Ensino de Ciências como "enculturação". In: CATANI, D. B; VICENTINI, P. P., (Orgs.). Formação e autoformação: saberes e práticas nas experiências dos professores. São Paulo: Escrituras, 2006.

CDC (CENTERS FOR DISEASE CONTROL AND PREVENTION). Health Literacy-A Public Health Priority. In: Health Literacy for Public Health Professionals. Disponível em <https://www.cdc.gov/healthliteracy/learn/index.html> Acesso em Jun, 2017.

CHAGAS, I. Literacia Científica. O Grande Desafio para a Escola. Comunicação apresentada no $1^{\circ}$ Encontro Nacional de Investigação e Formação, Globalização e Desenvolvimento Profissional do Professor. Escola superior de Educação de Lisboa, 2000.

CHASSOT, A. Alfabetização científica: questões e desafios para a educação. ljuí: Editora UNIJUÍ, 2000.

D'AMATO-KUBIET, LESLEE A. Nutrition literacy and demographic variables as predictors of adolescent weight status in a Florida county. [Dissertação de mestrado], Sumer term: Florida, 2013. 
DEBOER, G.E. Historical perspective in scientific literacy. 1997.

DEBOER. G. E. Scientific literacy: another look at its historical and contemporary meanings and its relationship to science education reform. Journal of Research in Science Teaching, Hoboken, v. 37, n. 6, p. 582-601, 2000.

DENUWARA, H. M. B. H.; GUNAWARDENA, N.P. Level of health literacy and factors associated with it among school teachers in an education zone in Colombo, Sri Lanka. BMC Public Health, v.17, n.631, p. 1-9, 2017.

DEPARTMENT OF HEALTH AND HUMAN SERVICES. Centers for Disease Control and Prevention. National Center for Health Statistics. Healthy people 2000: finale review. Hyattsville, Maryland, October 2001, DHHS Publication No. 01-0256, 2001.

DIAMOND, J.J. Development of a reliable and construct valid measure of nutritional literacy in adults. Nutrition Journal, v.6, n.5, 2007.

DIXEY R, HEINDL I; LOUREIRO I; PÉREZ-RODRIGO C, SNEL J; WARNKING P. Healthy Eating for Young People in Europe. A School-based Nutrition Education Guide. Copenhague: European Network of Health Promotiong Schools, 1999.

GABEL, L.L. The Development of a Model to Determine Perceptions of Scientific Literacy. Unpublished PhD thesis, Columbus, OH: Ohio State University, 1976.

GIBBS, H. D. Nutrition Literacy: Foundations and Development of an Instrument for Assessment. Faculty Scholarship. Family and Consumer Science, 2012.

GOOD, R; HAFNER, M; AND PEBBLES, P. Scientific understanding of sexual orientation: Implications for science education. American Biology Teacher, v. 62, n.5, p.326 - 330, 2000.

GRAÇA, S. C. Literacia científica: Conceitos e dimensões. In: AZEVEDO, F. SARDINHA, M.G. (Coord.). Modelos e práticas em literacia. Lisboa: Lidel, p.179-194, 2009.

HOLDBROOK, J.; RANNIKMAE, M. Nature of science education for enhancing scientific literacy. International Journal of Science Education. v.29, n.11, p.1347-1362, 2007.

HURD, P. Science literacy: It's meaning for American schools. Educational Leadership, 1958.

INSTITUTE OF MEDICINE: Health literacy: a prescription to end confusion. Washington DC: The National Academies, 2004.

KICKBUSCH, I.S. Health literacy: addressing the health and education divide. Health Promot Int., v. 16, n3, p.289-97, 2001.

LAUGKSCH, R. C. Scientific Literacy: A Conceptual Overview". Science Education, v. 84, n. 1, p. 71-94, 2000.

LAUGKSCH, R.C. A conceptual overview. Science Education, v.84, n.1, p.71-94, 1999.

LEAR, JOHN. The crisis in scientific education. American Newsletter. The new scientist, 26 june de 1958, v. 4, n, 84, p.238-284, 1958.

LEGER, L. Schools, health literacy and public health: possibilities and challenges. Health Promotion International. v.16, n. 2, p 197-205, 2001.

LORENZETTI, L.; DELIZOICOV, D. Alfabetização científica no contexto das séries inicias. Ensaio -Pesquisa em Educação em Ciências, v. 3, n. 1, jun. 2001. 
MAMEDE, M. E ZIMMERMANN, E., Letramento Científico e CTS na Formação de Professores para o Ensino de Física, trabalho apresentado no XVI SNEF - Simpósio Nacional de Ensino de Física, São Luís, 2007.

MILNER, I. Editorial New Zealand Science Teacher. NZASE Wellington, 2004.

MORTIMER, E. F. Construtivismo, mudança conceitual e ensino de ciências: para onde vamos? Investigações em ensino de ciências, 1(1), p. 20-39. 1996.

MORTIMER, E.F. MACHADO, A.H.A. A linguagem numa sala de aula de ciências. Presença Pedagógica, Belo Horizonte, v.11, n.2, p. 49-57, 1996.

NUTBEAM, D. Health literacy as a public health goal: achallenge for contemporary health education and communication strategies into the 21st century. Health Promot Int., p. 259267,2000

NUTBEAM, D. The evolving concept of health literacy. Social Science \& Medicine. v. 67 p. 2072-2078, 2008.

OCDE-ORGANIZAÇÃO PARA A COOPERAÇÃO E DESENVOLVIMENTO ECONÔMICO. Brasil no PISA 2015: análises e reflexões sobre o desempenho dos estudantes brasileiros. São Paulo: Fundação Santillana, 2016.

OSBORNE, J. Science education for the Twenty First Century. Eurasia Journal of Mathematics, Science and Technology Education, v. 3, n.3, p. 173-184, 2007.

PELLA, M. O., O'HEARN, G. T., GALE, C. G. Referents to

PÈRE-RODRIGO, C.; ARANCETA, J. Nutrition education in schools: experiences and challenges. European Journal of Clinical Nutrition, v.57, p. 82-\$85, 2003.

PETTERSEN, S.; KJØLLESDAL, J. G.; AARNES, S. B. Measuring nutrition literacy Paper presented at the 19th International Conference of Nutrition, Bangkok, Thailand, 2009.

ROBERTS, D. A. Scientific literacy. Towards a balance for setting goals for school, 1983.

RODRIGUES, L.P.F.; RONCADA, M. J. Educação nutricional no Brasil: evolução e descrição de proposta metodológica para escolas. Com. Ciências Saúde, v.,19, n.4, p.315-322, 2008.

RUDD, R.E.; ANDERSON, J.E.; NATH, C. Health literacy: an update of medical and public health literature. In: COMINGS, J.P.; GARNER, B.; SMITH, C. (Orgs.). Review of adult learning and literacy: connecting research policy and practice. Mahwah (New Jersey): Lawrence Erlbaum Associates Publishers, p. 175-204, 2007.

SAMPAIO, H. A. C.; SILVA, D. M. A; SABRY, M. O. D.; CARIOCA, A. A. F.; CHAYB, A. P. V. Nutrition literacy: performance of two Brazilian population groups. São Paulo: Rev. Soc. Bras. Alim. Nutr, v. 38, n. 2, p. 144-155, 2013.

SAMPAIO, H.A.C; et al. Avaliação do letramento em nutrição por dois métodos diagnósticos em brasileiros. Nutrición clínica y dietética hospitalaria, v. 34, n. 1, p.50-55, 2014.

SANTOS, W.L.P; MORTIMER, E.F. Tomada de Decisão para Ação Social Responsável no Ensino de Ciências. Ciência \& Educação, v.7, n.1, 95-111, 2001.

SASSERON, L. H. Alfabetização Científica no ensino Fundamental: Estrutura e Indicadores deste processo em sala de aula. Tese - Faculdade de Educação da USP, São Paulo, 2008. 
SASSERON, L. H.; CARVALHO, A. M. P. Construindo argumentação na sala de aula: a presença do ciclo argumentativo, os indicadores de Alfabetização Científica e o padrão de Toulmin. Ciência e Educação, v. 17, p. 97-114, 2011

SHAMOS. The Myth of Scientific Literacy. New Brunswick. N.J: Rutgers University Press, 1995. SHEN, B. S. P. Science Literacy. In: American Scientist, v. 63, p. 265-268, 1975.

SHOWALTER, V.M. What is united science education? Part 5. Program objectives and scientific literacy. Pris II, n.2, 1974.

SILK, K. J.; SHERRY J., WINNB; KEESECKERN, HORODYNSKI M.A; SAYIR A. Increasing nutrition literacy: testing the effectiveness of print, web site, and game modalities, Journal of Nutrition Education and Behavior, v. 40, p. 3-10, 2008.

TORRES, A.P.G; ZAPATA, J; PINTO, M.R. Una alfabetización científica tecnológica y cultural. Enseñanza de las ciencias: Bogotá, n. 11, p. 2009.

VELARDO, S. The Nuances of Health Literacy, Nutrition Literacy, and Food Literacy. Journal of Nutrition Education and Behavior, v.47, n.4, p. 385-389, 2015.

VIDGEN H.; GALLEGOS D. Defining food literacy and its components. Appetite, n.79, p.5059, 2014.

VIDGEN, H. Food literacy: key concepts for health and education. Routledeg: New York, p.245, 2016

ZABALA, A. A Prática Educativa. Como ensinar. Tradução Ernani F. da F. Rosa. Porto Alegre: ARTMED, 1998.

ZARCADOOLAS C, PLEASANT A, GREER D: Advancing health literacy: A framework for understanding and action. Jossey Bass: San Francisco, CA. 2006

ZOELLNER, J., CARR, D. H. Exploring nutrition literacy and knowledge among school nutrition, 2009. 